J. Lake Sci. (湖泊科学) , 2012, 24(6): 865-874

http: //www. jlakes. org. E-mail : jlakes@niglas.ac.cn

(C) 2012 by Journal of Lake Sciences

\title{
太湖梅梁湾富营养化主要驱动因子的多时间尺度分析”
}

\author{
李末, 秦伯强 \\ (中国科学院南京地理与湖泊研究所湖泊与环境国家重点实验室,南京 210008)
}

\begin{abstract}
摘 要: 采用太湖湖泊生态系统研究站太湖梅梁湾富营养化四种主要驱动因子叶绿素 a ( Chl. a)、水温、总磷 (TP)、总氮 浓度 1992-2010 年的监测数据, 利用小波变换方法, 分析了四种因子不同时间尺度上的时间格局特征. 结果表明, 四种驱 动因子在年际和年代际尺度上都具有清晰的多时间尺度特征, 主周期各不相同, 但 Chl. a 和 TP 浓度的小波实部变化趋势 具有较高的一致性. 本文分析了各因子在不同时间尺度上的强弱分布以及高低交替, 并在此基础上从时间尺度的角度预 测出在未来 $3 \sim 5$ 年内, 太湖梅梁湾湖区的富营养化程度将保持在一个比较平稳的水平, 为太湖富营养化的认识和治理提 供更加可靠的科学依据.
\end{abstract}

关键词: 太湖;梅梁湾;富营养化; 小波分析;多时间尺度

\section{Multiple temporal scale analysis of main driving factors of eutrophication in Meiliang Bay, Lake Taihu}

\section{Wei \& QIN Boqiang}

( State Key Laboratory of Lake Science and Environment, Nanjing Institute of Geography and Limnology, Chinese Academy of Sciences, Nanjing 210008, P. R. China)

\begin{abstract}
Concentration of chlorophyll-a (Chl. a), total phosphorus (TP), total nitrogen and water temperature are the four main driving factors resulting in eutrophication in Meiliang Bay, Lake Taihu. Based on the observation data in the period of $1992-$ 2010 , the temporal pattern characteristics of the four factors in different temporal scales were analyzed respectively using wavelet analysis. Results showed that the temporal patterns of the four factors were obvious in the inter-annual and inter-decadal scales and the primary periods were different, but the real parts of wavelet coefficients of Chl. a and TP were consistent. The distributions and the periodic oscillation were analyzed and it was predicted that the eutrophication condition in Meiliang Bay would keep stable in the future 3-5 years. This method will provide reliable scientific evidence to the management of Lake Taihu.
\end{abstract}

Keywords: Lake Taihu; Meiliang Bay; eutrophication; wavelet analysis; multiple temporal scale

太湖是我国第三大淡水湖泊, 是长江中下游经济发达地区重要的水源地, 但是自 1980s 以来, 太湖频繁 暴发蓝藻水华, 富营养化问题日益严重. 在人类活动的影响下, 氮、磷等营养物质大量进人太湖, 引起藻类及 其它浮游生物迅速繁殖, 水体透明度和溶解氧含量下降, 水质恶化, 鱼类及其它生物死亡, 生物多样性减少, 水体生态系统和功能受到严重破坏. 根据前人的研究 ${ }^{[-3]}$, 浮游藻类的生长是导致湖泊富营养化的关键因 素, 而浮游藻类的生长是由营养盐、水文气象条件等因素共同控制的, 反映生物类别及数量的指标叶绿素 a (Chl. a), 反映营养盐水平的指标总氮 (TN)、总磷 ( TP), 以及反映植物生长环境的指标水温 $(\mathrm{T})$, 是驱动太 湖富营养化的主要因子. 对这些因子进行长期连续监测, 对其时间序列进行研究与分析, 可以认识因子系统 的固有特性和时间格局特征, 了解水体的生态系统状况和富营养化水平, 并进一步推断其在将来的变化和 行为, 对于水体富营养化的控制和保障水质安全具有重要意义.

目前, 对于太湖富营养化驱动因子的研究, 大多来源于对实测资料时间序列的统计分析或者时域分析, 在时间域内对动态过程进行研究. 比如, 陈宇炜等 ${ }^{[4]}$ 以太湖梅梁湾 $1992-1999$ 年的监测资料为基础, 选取

* 国家自然科学基金项目 (40801200) 和国家杰出青年基金项目 (40825004) 联合资助. $2011-11-15$ 收稿; $2012-$ 03-13收修改稿. 李未, 女, 1980 年生, 博士, 助理研究员; E-mail : liwei@ niglas. ac. cn. 
水温等 15 项环境理化因子与 Chl. a 进行逐步回归分析, 预测了梅梁湾藻类生物量的变化情况; 焦锋等 ${ }^{[5]}$ 对 2000-2002 年太湖梅梁湾年报监测数据进行二元变量相关性分析, 认为磷是梅梁湾富营养化的主要限制性 因子, 水温与光照是富营养化的重要影响因素; 朱广伟 ${ }^{[6]}$ 根据太湖湖泊生态系统研究站 $2005-2006$ 年的监 测数据, 结合历史监测记录, 认为自 2000 年以来, 太湖的富营养化状况有加重趋势; 申哲民等 ${ }^{[7]}$ 对太湖 1980-2008年 TN、TP、Chl. a、气温和水温的年平均数据进行研究, 应用修正的 Monod 生长模型定量研究 了太湖藻类生物量与营养元素和温度的关系; 龚绍琦等 ${ }^{[8]}$ 利用太湖 $1998-2004$ 年的叶绿素月监测数据, 通过研究数据之间的自相关结构, 建立了 ARMA 时间序列模型, 对不同湖区的叶绿素浓度进行动态模拟 和预测. 然而对于太湖富营养化驱动因子长时间序列的时间格局特征和多层次演变规律的研究却鲜有 报道.

由于驱动因子的时间序列中包含了自然和人类干扰等多种综合因素, 一般属于非平稳序列, 不仅具有 趋势性、周期性等特征, 还存在随机性、突变性以及 “多时间尺度” 结构, 具有多层次演变规律 ${ }^{[9]}$, 仅靠时域分 析无法揭示隐藏在时间序列中的多种变化周期, 以及系统在不同时间尺度中的变化趋势和突变特征. 为了 对太湖富营养化主要驱动因子的周期模式及时间格局特征有更加清楚的认识, 为太湖富营养化的治理提供 更加可靠的科学依据, 本文采用时域一频域联合分析的小波变换方法, 对 1992-2010 年近 $20 \mathrm{a}$ 来太湖梅梁 湾 Chl. a 浓度、水温、TP 和 TN 浓度时间序列的变化特征和规律进行分析, 明确各因子的周期特征和突变点, 揭示其在不同时间尺度上的结构和异常变化规律.

\section{1 小波分析方法简介}

小波分析 (Wavelet analysis) 是 1980s 初发展起来的一种具有时间-频率多分辨率分析 (Multi-resolution analysis) 功能的新方法, 在时频两域都具有表征时间序列局部特征的能力, 在低频部分具有较高的频率分辨 率和较低的时间分辨率, 在高频部分具有较高的时间分辨率和较低的频率分辨率, 具有对时间序列的自适 应性, 被誉为 “数学显微镜” ${ }^{[10-11]}$, 已经开始越来越多地被应用于水文、气温、降水、水质等非平稳时间序列的 多尺度分析和研究中, 并获得了较好的结果 ${ }^{[12-20]}$.

\section{1 小波函数}

小波函数 $\psi(t)$ 是指具有震荡特性, 能够迅速衰减到零的一类函数, 即:

$$
\int_{-\infty}^{+\infty} \psi(t) \mathrm{d} t=0
$$

式中, $\psi(t)$ 为母小波 (Mother Wavelet) 函数,通过尺度的伸缩和时间轴上的平移, 可以得到一个小波序列:

$$
\psi_{a, b}(t)=|a|^{-1 / 2} \psi\left(\frac{t-b}{a}\right), a, b \in R, a \neq 0
$$

式中, $\psi_{a, b}(t)$ 为子小波, $a$ 为尺度因子, 反映小波的周期长度; $b$ 为时间因子, 反映时间上的平移.

不同的母小波函数可以得到不同的小波序列. 在大气、水文及环境等领域的应用中, 常用的小波函数有 实数 Mexican hat 小波、Marr 小波和复数 Morlet 小波等 ${ }^{[12-20]}$. 实数小波变换可以给出时间序列变化的振幅和 正负; 而复数小波变换系数有模和实部两个重要变量, 模的大小表示特征时间尺度信号的强弱, 实部可表示 不同特征时间尺度信号在不同时间的强弱和位相两方面的信息, 较实数小波更能真实反映各尺度周期性的 大小及这些周期在时域中的分布, 更有利于对时间序列的分析. 因此本文选取 Morlet 连续复数小波函数 $\psi(t)=\mathrm{e}^{i \omega_{\sigma} t} \mathrm{e}^{-t^{2} / 2}, \omega_{0}=6$ 对时间序列进行小波变换.

\section{2 小波变换}

对于给定的小波函数, 对实际工作中通过观测得到的某时间序列数据 $f(k \Delta t), k=1,2, \cdots, N, \Delta t$ 为取样 时间间隔,其离散形式的连续小波变换为:

$$
W_{f}(a, b)=|a|^{-1 / 2} \Delta t \sum f(k \Delta t) \bar{\psi}\left(\frac{k \Delta t-b}{a}\right)
$$

式中, $W_{f}(a, b)$ 称为小波系数, $\bar{\psi}(t)$ 为 $\psi(t)$ 的复共轭函数. 
在实际应用中, 由小波变换方程得到小波系数 $W_{f}(a, b), W_{f}(a, b)$ 随 $a$ 和 $b$ 变化, 可以作出以 $b$ 为横坐 标, $a$ 为纵坐标的二维等值线图,通过此图得到时间序列的小波特征.

\section{3 边界效应的消除}

为了消除时间序列起始点与结束点数据 “边界效应” 对小波信息的影响, 利用对称延拓法 ${ }^{[21]}$ 对数据进 行延伸, 延拓部分的数据与延拓端数据对称, 延拓时间序列为:

$$
\cdots, f(2 \Delta t), f(\Delta t), \vdots f(\Delta t), f(2 \Delta t), \cdots, f(N \Delta t), \vdots f(N \Delta t), f((N-1) \Delta t), \cdots
$$

\section{4 小波方差}

将小波系数的平方在 $b$ 域上积分, 就可得到小波方差, 即:

$$
\operatorname{Var}(a)=\int_{-\infty}^{\infty}\left|W_{f}(a, b)\right|^{2} \mathrm{~d} b
$$

小波方差随尺度 $a$ 的变化过程, 称为小波方差图, 能反映信号波动的能量随尺度 $a$ 的分布. 因此, 小波 方差图可用来确定信号中不同尺度扰动的相对强度和存在的主要时间尺度,即主周期.

\section{2 数据来源与处理}

自 1992 年开始, 太湖湖泊生态系统研究站 (TLLER) 开始以梅梁湾为主对太湖的生态环境进行常规监 测和资料累积, 并在近几年陆续扩展到全太湖 32 个点位, 监测点位分布见图 1. 其中梅梁湾内 $1^{*}$ 点从 1992 年起就逐月对 Chl. a、TN、TP 等各生态指标进行观测, 样品的采集、分析均按照《湖泊生态系统观测方法》进

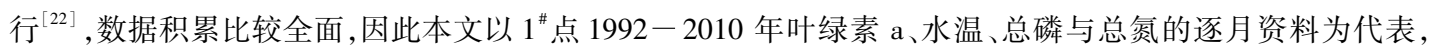
对太湖梅梁湾富营养化的主要驱动因子进行多时间尺度分析.

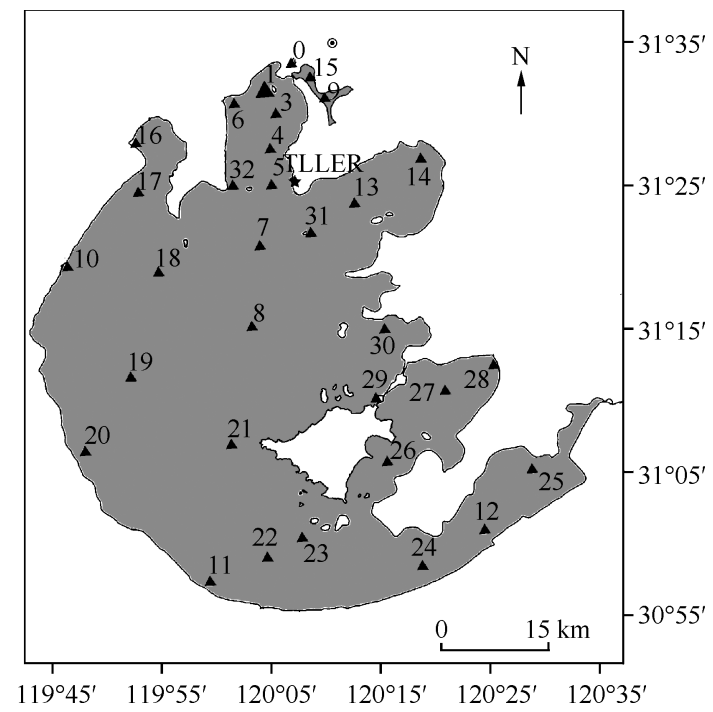

图 1 太湖监测点位分布

Fig. 1 The distribution of monitoring sites in Lake Taihu

$1^{\#}$ 点 1992-2010 年 Chl. a 、水温、TP 和 TN 的时间序列及多年月平均曲线可看出, 各指标呈现出明显的 季节变化和周年变化 (图 2). 其中 Chl. a、水温和 TP 的变化趋势是夏季高、冬季低, 每年的最高值一般发生 在 $7 、 8$ 月份, 最低值发生在 12 月份或次年 1 月份; 而 TN 的变化趋势是冬季高、夏季低, 每年的最低值一般 发生在 $8 、 9$ 月份,最高值发生在 $1-3$ 月份.

在小波变换之前, 首先计算各因子数据 1992-2010 年的年平均序列, 并对年平均序列进行中心化处 理, 即: 

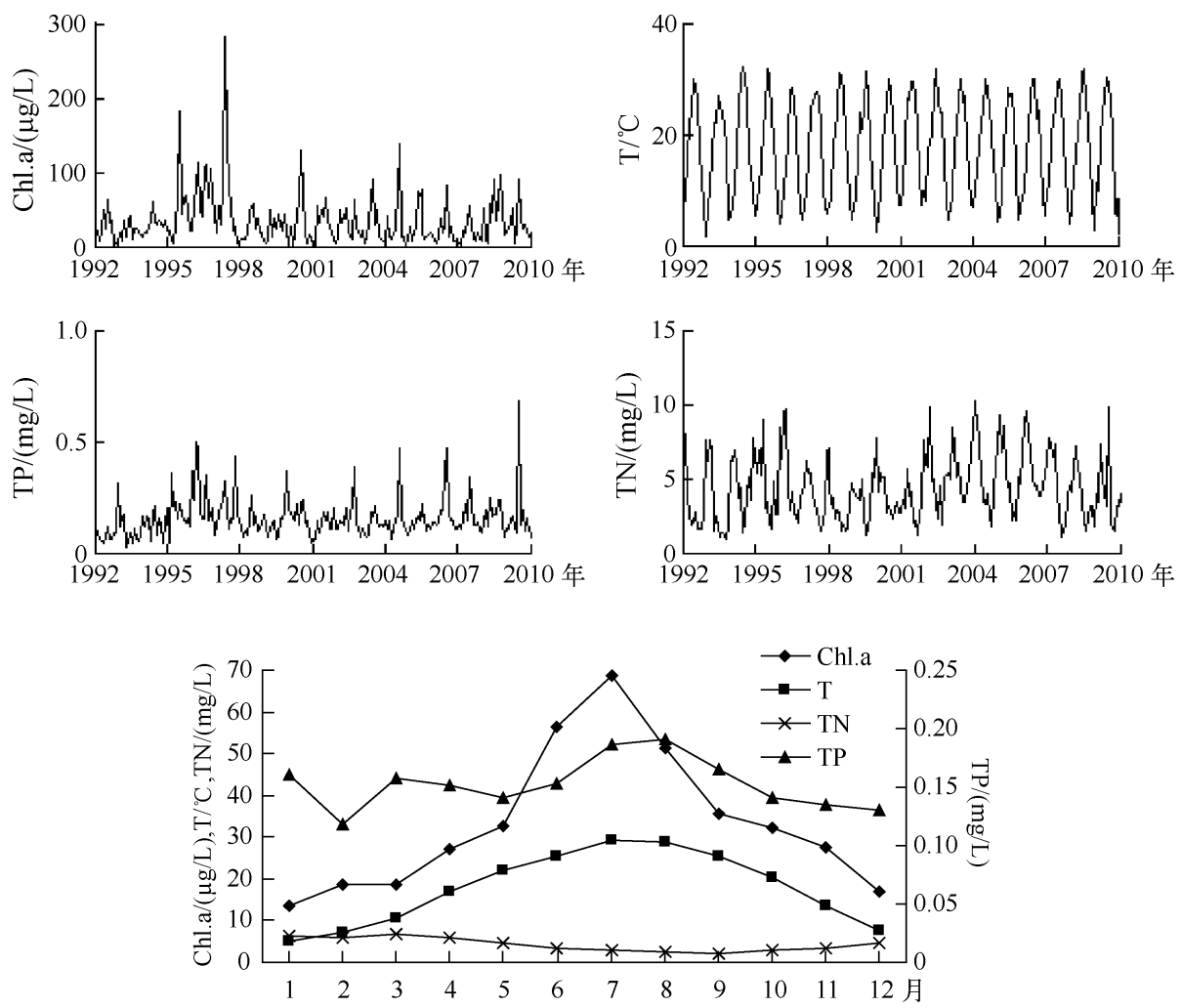

图 2 梅梁湾 $1^{\#}$ 点 Chl. a、水温、TP、TN 1992-2010 年时间序列曲线及多年月平均曲线

Fig. 2 The time series and monthly-averaged variations of chlorophyll-a, water temperature, total phosphorus and total nitrogen at $1^{\#}$ site in Meiliang Bay from 1992 to 2010

$$
x_{t}^{\prime}=\left(x_{t}-\bar{x}\right) / s, t=1,2, \cdots, n
$$

式中, $\bar{x}$ 和 $s$ 分别是年平均序列的平均值和标准差. 然后对中心化的样本值进行 Morlet 小波变换, 分别计算 出小波系数的模平方、实部和小波方差, 画出小波系数时频分析图分析驱动因子的多时间尺度演变特征和 突变特征.

\section{3 富营养化驱动因子的多时间尺度分析}

\section{1 Chl. a 浓度}

年平均 Chl. a 浓度序列 Morlet 小波变换模平方的时频分布图可以看出, Chl. a 浓度在不同时间段不同频 率周期性变化的强弱分布 (图 3a). 在年 (小于 3 a) 时间尺度上, Chl. a 浓度没有表现出明显的时间格局变 化. 在年际(小于 $10 \mathrm{a}$ ) 时间尺度上,5 7 a 间,1992-1998 年的高频波动表现最强烈, 振荡中心出现在 1995 年, 振荡中心的时间尺度为 $6 \mathrm{a}$ 左右; 在 2003-2006 年和 2007-2010 年也出现了这一波段的扰动, 但强度 较弱,中心分别出现在 2005 年和 2009 年; 7 9 a 间, 1992-1997 年的波动很强烈,振荡中心出现在 1992 年. 在年代际 (大于 $10 \mathrm{a}$ ) 时间尺度上, 1992-2002 年前后的波动表现较强烈, 振荡中心的时间尺度为 $14 \sim 15 \mathrm{a}$ 之间.

年平均 Chl. a 浓度序列 Morlet 小波变换实部的时频分布图可以看出, Chl. a 浓度在不同频率周期性变化 强弱分布、突变线以及位相结构 (图 3b). 在 $15 \mathrm{a}$ 以上年代际时间尺度上, Chl. a 浓度经历了低一高一低一高的 循环交替,其中 1995 年以前、2000-2005 年 Chl. a 浓度相对较低, 1995-1999 年、2006-2010 年 Chl. a 浓度 相对较高,而且 2006-2010 年等值线尚未闭合,说明 2010 年以后一段时间内 Chl. a 浓度仍将偏高. 在 $10 \sim$ 
$13 \mathrm{a}$ 时间尺度上, Chl. a 浓度经历了低一高-低一高-低 5 个循环交替; 在 $5 \sim 7$ a 时间尺度上, 则有更多的 Chl. a 浓度相对偏高期和偏低期的循环交替, 突变点增多.

Chl. a 浓度序列周期为 $6 a 、 10 \mathrm{a}$ 和 $15 \mathrm{a}$ 的小波变换实部变化过程可以准确地显示出, 在不同的时间尺 度下年平均 Chl. a 浓度的高低变化和突变点 (图 3c). 例如在 $15 \mathrm{a}$ 特征时间尺度上, Chl. a 浓度经历了 2 个周 期的低一高变化, 平均变化周期为 $10 \mathrm{a}$ 左右; 而在 $6 \mathrm{a}$ 特征时间尺度上, Chl. a 浓度的平均变化周期为 $5 \mathrm{a}$ 左 右, 大约经历了 4 个高-低转换期.
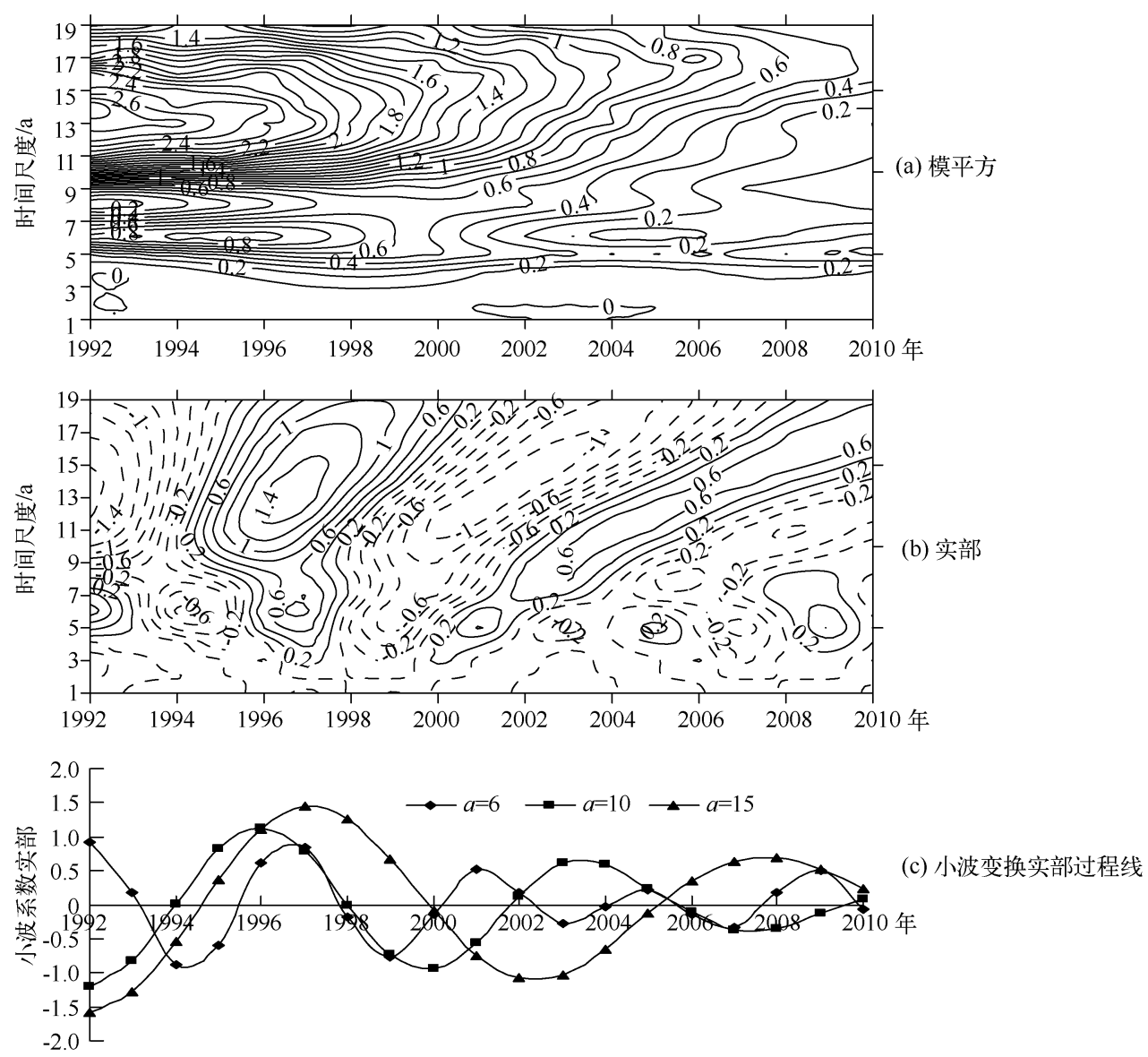

图 3 Chl. a 浓度小波变换图

Fig. 3 Wavelet analyses of Chl. a concentration

\section{2 水温}

年平均水温序列小波变换模平方的时频分布图可以看出,水温在 3 6 a 时间尺度上, 1995-2003 年的高频波动表现最强烈, 振荡中心出现在 1999 年, 振荡中心的时间尺度为 $4 \mathrm{a}$ 左右. 振荡中心的时 间尺度为 $7 \mathrm{a}$ 左右, 以 1993-2002 年的波动最为强烈, 振荡中心在 1997 年; 2002-2010 年以振荡中 心时间尺度为 $8 \mathrm{a}$ 的波动最强烈, 振荡中心在 2007 年. 在 1999-2007 年, 出现了以振荡中心时间尺度 为 $11 \mathrm{a}$ 左右的波动, 但强度较弱. 振荡在 $15 \mathrm{a}$ 以上的波动, 在 2001 年以后表现较明显, 强度较弱 (图 4a).

年平均水温序列小波变换实部的时频分布图可以看出, 在 $15 \mathrm{a}$ 以上年代际时间尺度上, $\mathrm{T}$ 经历了高低一高-低的循环交替, 其中 1996 年以前、2003-2006 年 T 相对较高, 1997-2002 年、2007-2010 年 T 相对 较低, 而且 2007-2010 年等值线仍未闭合, 说明 2010 年以后一段时间内 $\mathrm{T}$ 仍将偏低. 在 $11 \sim 13$ a 时间尺度 
上, $\mathrm{T}$ 经历了低一高-低-高-低 5 个循环交替; 在 $7 \sim 10 \mathrm{a}$ 时间尺度上, $\mathrm{T}$ 经历了低一高-低-高-低-高-低 7 个循 环交替; 对应 $2 \sim 4 \mathrm{a}$ 小时间尺度的 $\mathrm{T}$ 变化, 则有更多的高温期和低温期的循环交替, 突变点增多, 振荡中心 的时间尺度为 $3 \mathrm{a}$ 左右 (图 $4 \mathrm{~b}$ ).

$\mathrm{T}$ 序列周期为 $4 \mathrm{a}, 8 \mathrm{a}$ 和 $17 \mathrm{a}$ 的小波变换实部变化过程可以看出, 年平均水温的多时间尺度变化表现十 分明显, 水温偏高期和偏低期交替出现, 突变点位置及分布十分清晰 (图 4c).
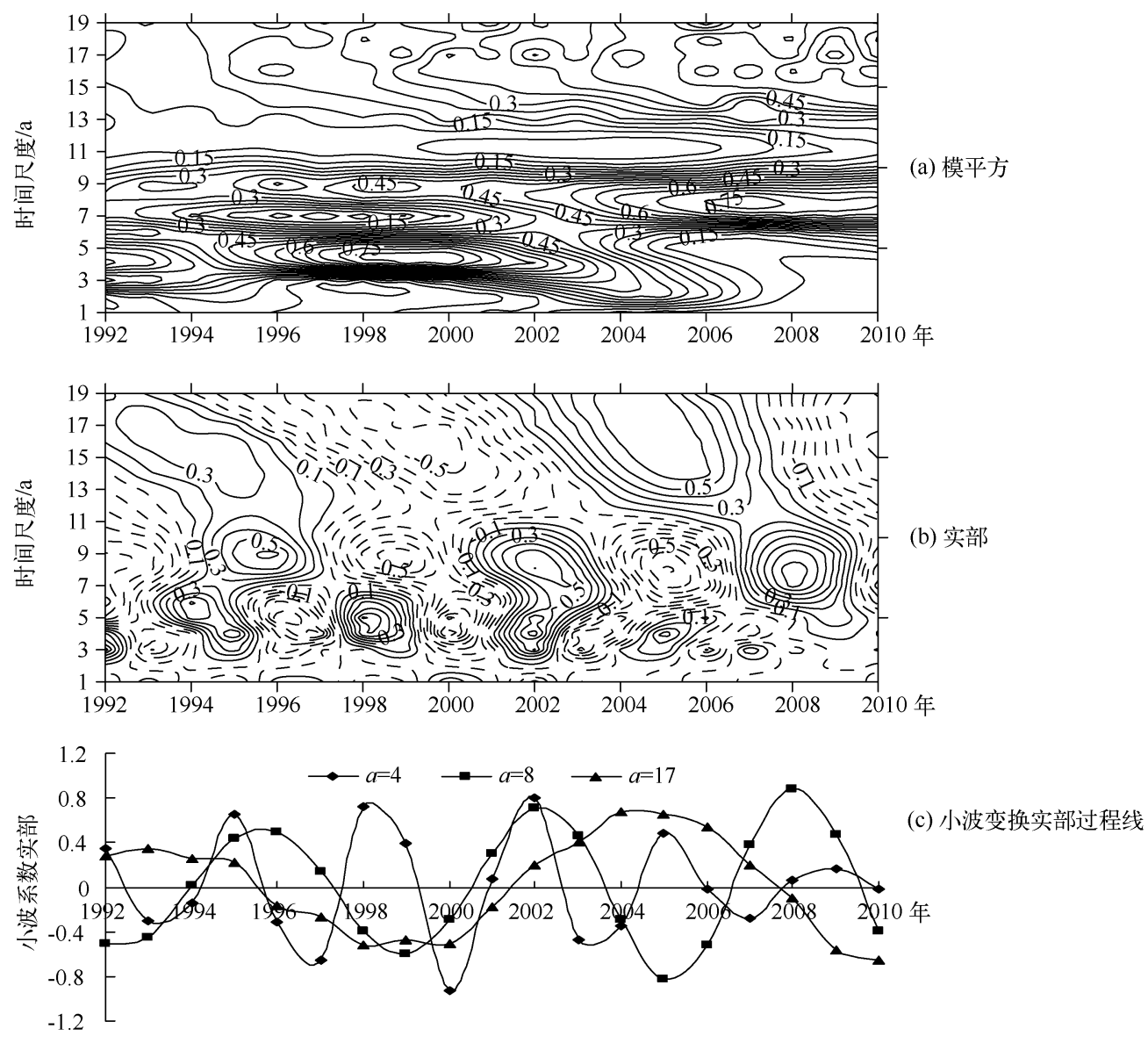

图 4 水温小波变换图

Fig. 4 Wavelet analyses of water tempreture

\section{3 TP 浓度}

年平均 TP 浓度序列小波变换模平方的时频分布图可以看出, TP 浓度在年代际 $11 \sim 15$ a 时间尺度上, 1992-2002 年的波动表现最为强烈, 振荡中心的时间尺度为 $14 \mathrm{a}$ 左右, 其余波动的强度较弱 (图 5a). 在 15 a以上年代际时间尺度上, TP 浓度经历了低一高-低一高的循环交替, 其中 1994 年以前、1999-2004 年浓度 相对较低, 其余年份浓度相对较高, 而且 2005-2010 年等值线已经闭合, 说明 2010 年以后一段时间内 TP 浓 度又将开始偏低. 在 $10 \sim 15 \mathrm{a}$ 时间尺度上, TP 浓度经历了低一高-低一高-低 5 个循环交替; 在 $8 \sim 9 \mathrm{a}$ 年际时 间尺度上, TP 经历了低一高一低一高一低一高-低 7 个循环交替; 对应 $3 \sim 5 \mathrm{a}$ 的 TP 浓度变化, 则有更多的高浓度 期和低浓度期的循环交替. 而 3 a 以下年尺度上, TP 浓度时间格局变化不明显(图 $5 \mathrm{~b}$ ).

$\mathrm{TP}$ 浓度序列周期为 $3 \mathrm{a} 、 7 \mathrm{a} 、 10 \mathrm{a}$ 和 $15 \mathrm{a}$ 的小波变换实部变化过程可以看出, 年平均 TP 浓度在不同的 时间尺度下存在不同的平均周期和高-低浓度变化特征,多时间尺度特征十分明显(图 $5 \mathrm{c}$ ). 

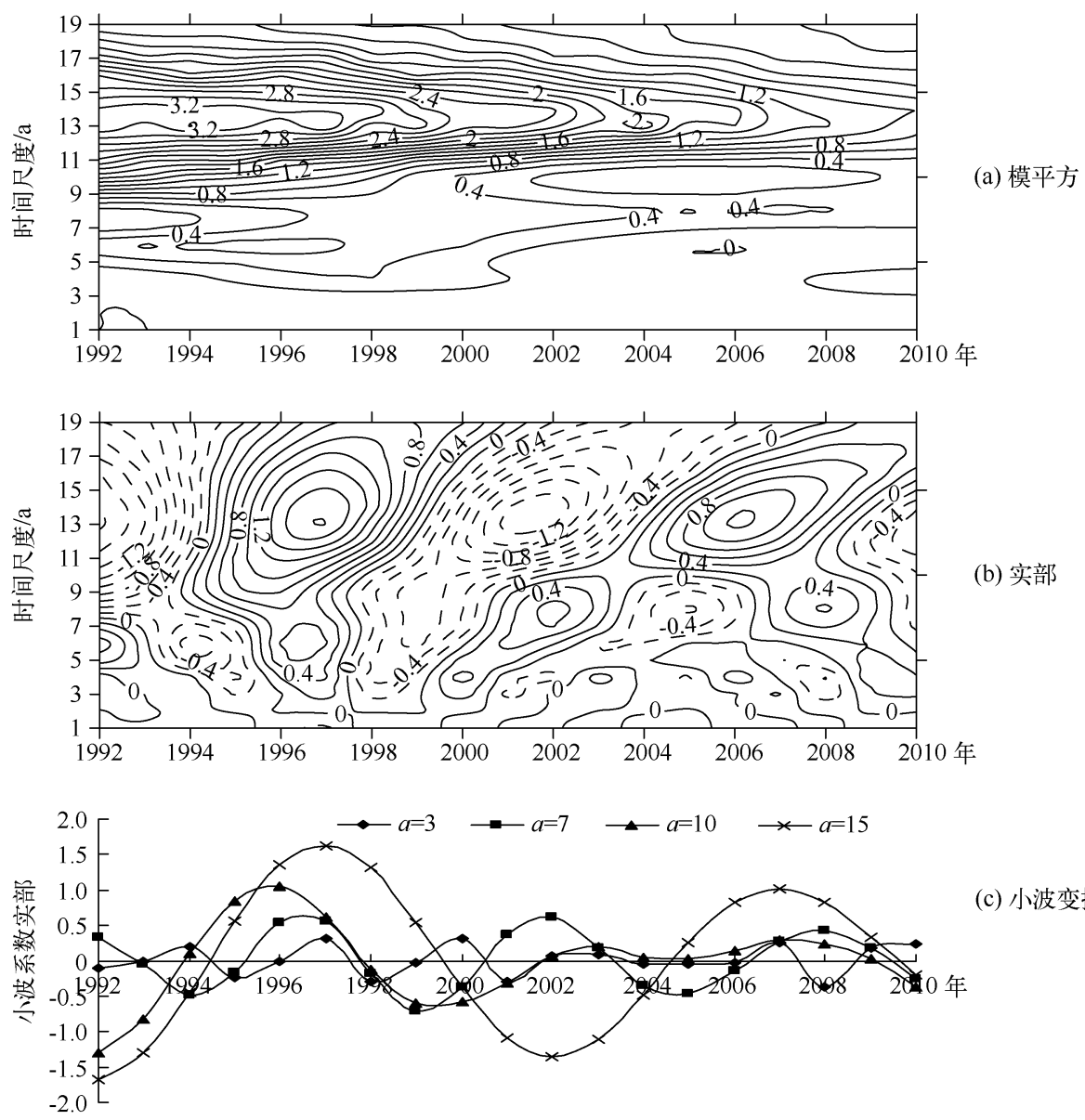

(c) 小波变换实部过程线

图 $5 \mathrm{TP}$ 浓度小波变换图

Fig. 5 Wavelet analyses of TP concentration

\section{3. $4 \mathrm{TN}$ 浓度}

年平均 $\mathrm{TN}$ 浓度序列小波变换模平方的时频分布图可以看出, TN 浓度在 $7 \sim 10 \mathrm{a}$ 年际时间尺度上, 19982010 年的波动表现最为强烈, 振荡中心出现在 2005 年, 振荡中心的时间尺度为 $9 \mathrm{a}$ 左右. 在 $11 \sim 13 \mathrm{a}$ 的年 代际时间尺度上,1992-1994 年的波动较明显,但强度较弱,振荡中心在 1992 年(图 6a).

在 15 a 以上年代际时间尺度上, TN 浓度经历了低-高-低的循环交替, 其中 2000-2006 年浓度相对较 高, 其他年份浓度相对较低, 而且 2007-2010 年等值线仍未闭合, 说明 2010 年以后一段时间内 TN 浓度仍 将偏低 (图 6b). 在 11 13 a 年代际时间尺度上, TN 浓度经历了高一低一高-低 4 个循环交替; 在 7 10 a 年际 时间尺度上, TN 经历了低-高-低一高-低 5 个循环交替; 对应 $1 \sim 3 \mathrm{a}$ 年尺度的 TN 浓度变化, 则在 1996 年以 后变化比较明显,对应着 $\mathrm{TN}$ 浓度的季节变化特征(图 6b).

$\mathrm{TN}$ 浓度序列周期为 $2 \mathrm{a} 、 7 \mathrm{a} 、 12 \mathrm{a}$ 和 $17 \mathrm{a}$ 的小波变换实部变化过程可以看出,年平均 TN 浓度在不同的 时间尺度下存在不同的平均周期和浓度高-低变化特征,多时间尺度特征十分明显 (图 $6 \mathrm{c}$ ).

\section{5 小波方差分析}

Chl. a 浓度的小波方差图中存在 2 个较为明显的峰值, 依次对应着 $6 \mathrm{a}$ 和 $15 \mathrm{a}$ 的时间尺度. 其中对 应着 $15 \mathrm{a}$ 时间尺度的峰值较大, 说明 $15 \mathrm{a}$ 左右的周期震荡较强, 为 Chl. a 浓度变化的第一主周期; $6 \mathrm{a}$ 时间尺度对应着第二峰值, 为 Chl. a 浓度变化的第二主周期, 这 2 个周期的波动控制着 Chl. a 浓度在 

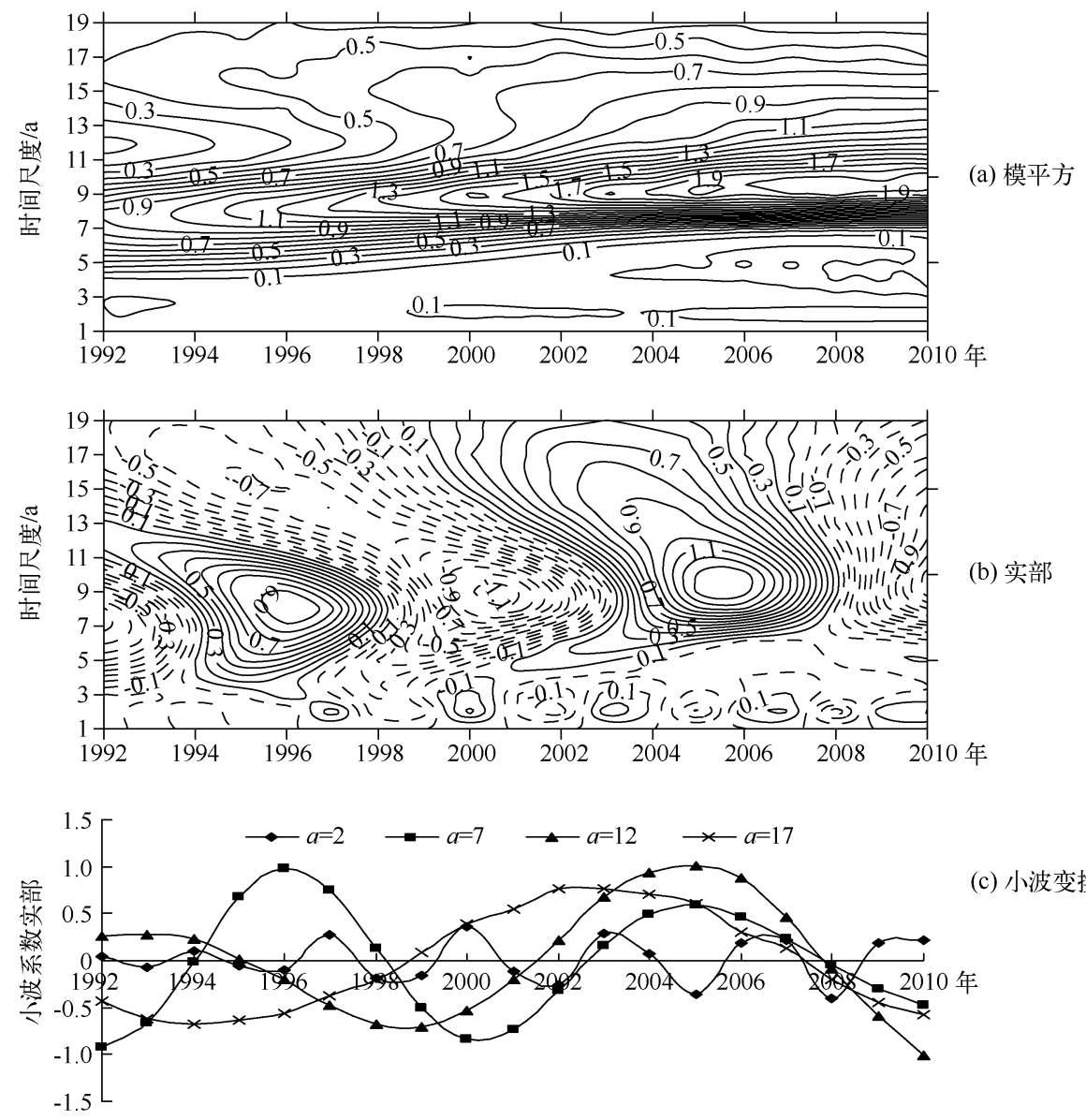

(c) 小波变换实部过程线

图 $6 \mathrm{TN}$ 浓度小波变换图

Fig. 6 Wavelet analyses of TN concentration

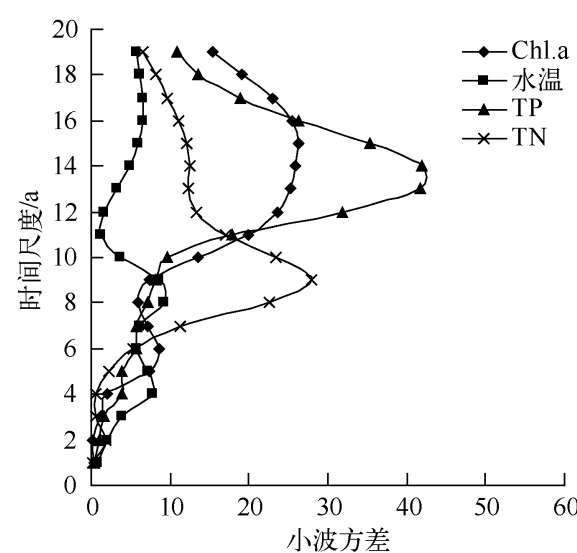

图 7 叶绿素 a、水温、总磷、总氮的小波方差

Fig. 7 Wavelet variance of chlorophyll-a, water temperature, total phosphorus and total nitrogen
整个时间域内的变化特征. 同样, 水温序列存在 $4 \mathrm{a}$ 、 $8 \mathrm{a}$ 和 $16.5 \mathrm{a}$ 三种不同时间尺度的准主周, 其 中以 $8 \mathrm{a}$ 的波动最为强烈; TP 序列存在 $4 \mathrm{a} 、 6 \mathrm{a}$ 和 $13.5 \mathrm{a}$ 三种不同时间尺度的准主周, 其中以 $13.5 \mathrm{a}$ 的高频波动最为强烈; TN 序列存在 $2 \mathrm{a}$ 和 $9 \mathrm{a}$ 两种 不同时间尺度的准主周, 其中以 $9 \mathrm{a}$ 的高频波动最 为强烈 (图 7 ).

\section{4 讨论}

根据以上小波变换方法分别得到了太湖梅梁湾富 营养化四种主要驱动因子 (Chl. a 浓度、水温、TP 和 TN 浓度) 的多时间尺度变化特征, 通过分析可以看出, 四 种驱动因子都具有明显的多时间尺度特征, 小尺度的 周期振荡嵌套于大、中尺度的周期振荡之中. 大尺度 (年代际尺度) 的周期振荡特征反映了各因子在整个时 间域内的变化特征, 如趋势性特征; 而中、小尺度 (年尺 
度和年际) 的周期振荡则聚焦各因子的精细结构,如浓度的高低变化.

四种驱动因子精细的时间格局特征不尽相同, 主周期也不同, Chl. a 浓度与 TP 浓度是以年代际时间尺 度的主周期为主控制其在整个时间域内的变化, 水温和 TN 浓度则是以年际时间尺度的主周期为主控制其 在整个时间域内的变化. 这些因子时空格局的形成, 应该是对同时期某些影响因素 (如水动力过程或气候变 化) 的响应,在以后的工作中,将结合与之相关的水文与环境要素时间序列,做进一步的研究.

由于某些因子不具有年尺度上的时间格局变化, 分别以时间尺度 $a=5 、 10 、 15$ 为代表对年际与年代际 尺度上四种因子在同一时间尺度下的小波变换实部变化过程进行对比发现, 无论是在年际尺度还是在年代 际尺度上, Chl. a 和 TP 浓度的小波实部变化过程的趋势一致性较高, 浓度值发生高低转换的年份也基本相 同, 表明二者的周期特征之间存在较强的相关性, 而水温和 TN 的实部变化趋势与 Chl. a 和 TP 存在不同程 度的相位差, 与 Chl. a 和 TP 浓度不具有明显的相关关系. 而且, 2008-2010 年处于小波实部突变年份, 四种 因子都已经或即将进人小波系数实部的负值区, 即四种因子的值都处于相对较低的水平, 由此可以预见, 在 目前太湖水环境综合治理力度较强的状态下, 今后 $3 \sim 5$ a 内, 太湖梅梁湾湖区的富营养化程度将保持在一 个比较平稳的水平, 富营养化程度突然加剧的可能性较低. 但是 $3 \sim 5 \mathrm{a}$ 之后, 四种因子的小波系数又将进人 高值区, 势必会影响到太湖梅梁湾富营养化的程度, 因此太湖富营养化的治理是一个需要长期坚持的过程 (图 8).
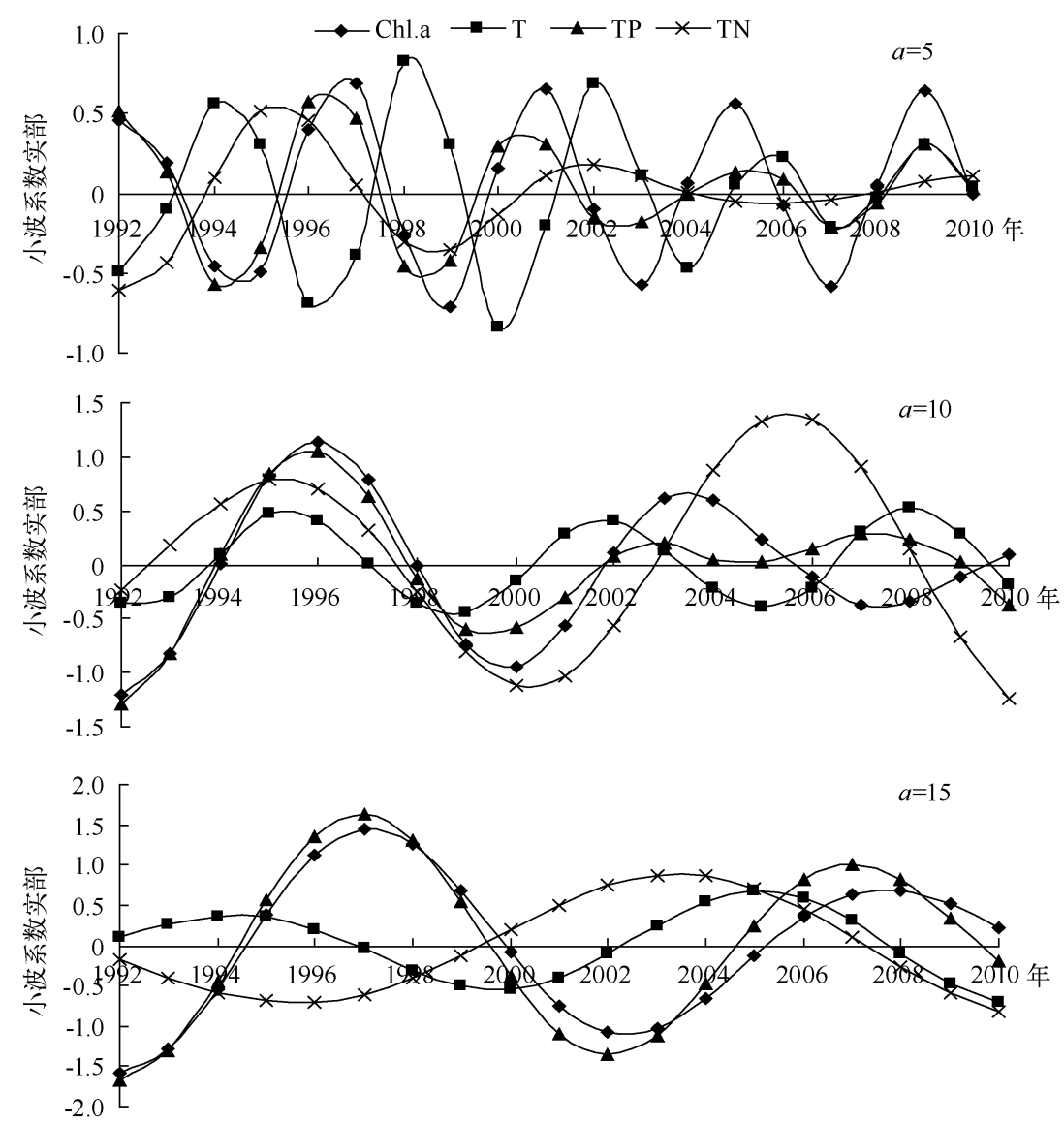

图 8 四种因子在相同时间尺度下小波变换实部过程线对比

Fig. 8 Real part of wavelet coefficients of the four factors at the same temporal scale 


\section{5 结论}

本文利用小波变换的方法对驱动太湖梅梁湾富营养化的四种因子 1992-2010 年的年均时间序列进行 分析, 得到四种因子不同时间尺度上的时间格局特征, 清晰地给出了各种时间尺度的强弱分布情况以及突 变点, 分析出各因子的主要周期, 并从时间尺度的角度对太湖梅梁湾湖区未来富营养化的演变趋势进行定 性的估计和预测, 为解释太湖富营养化进程提供了一个新的理论依据.

致谢: 感谢太湖湖泊生态系统研究站提供 1992-2010 年的监测数据.

\section{6 参考文献}

[ 1 ] Jorgensen SE. Application of ecology inenvironmental management. Boca Raton: CRC Press, 1983.

[2] 李一平,逢 勇,吕 俊等. 太湖富营养化的驱动因子分析. 河海大学学报: 自然科学版, 2004,32 (6):644-647.

[ 3 ] 官 涤,任伊滨,李 菁. 环境因子对水华暴发的影响研究. 环境科学与管理,2011,36(8):55-59.

[ 4 ] 陈宇炜, 秦伯强, 高锡云. 太湖梅梁湾藻类及相关环境因子逐步回归统计和蓝藻水华的初步预测. 湖泊科学, 2001, $13(1): 63-71$.

[ 5 ] 焦 锋,李 新. 太湖梅梁湾富营养化相关问题探讨. 环境污染与防治,2005,27(3):214-217.

[ 6 ] 朱广伟. 太湖富营养化现状及原因分析. 湖泊科学, 2008,20(1):21-26.

[ 7 ] 申哲民,张 涛,马 晶等. 富营养化与温度因素对太湖藻类生长的影响研究. 环境监控与预警, 2011,3(3):14.

[ 8 ] 龚绍琦, 黄家柱, 李云梅等. 应用时间序列分析法对太湖叶绿素-a 含量的动态研究. 海洋与湖沼, 2008, 39 (6): 591-598.

[9] 王黎明,王 连,杨 楠. 应用时间序列分析. 上海:复旦大学出版社,2009.

[10］崔锦泰. 小波分析导论. 西安:西安交通大学出版社,1995.

[11] 胡昌华, 李国华, 周 涛. 基于 MATLAB7. X 的系统分析与设计一一波分析. 西安: 西安电子科技大学出版 社, 2008 .

[12] 王文圣,丁 晶, 向红莲. 小波分析在水文学中的应用研究及展望. 水科学进展, 2002,13(4):515-520.

[13] 王文圣,丁 晶, 向红莲. 水文时间序列多时间尺度分析的小波变换法. 四川大学学报: 工程科学版, 2002,34(6): 14-17.

[14] 王文圣,丁 晶,衡 形等. 水文序列周期成分和突变特征识别的小波分析法. 工程勘察, 2003, (1):32-35.

[15] 牛存稳,张利平,夏 军. 华北地区降水量的小波分析. 干旱区地理,2004,27(1):66-70.

[16] Kang SJ, Lin H. Wavelet analysis of hydrological and water quality signals in an agricultural watershed. Journal of Hydrology, 2007, 338: 1-14.

[17] 江学顶, 夏北成, 郭 讶等. 广州城市热岛空间分布及时域-频域多尺度变化特征. 应用生态学报, 2007,18(1): 133-139.

[18］姜世中, 梁 川. 龙川江年输沙率时间序列的小波特征. 哈尔滨工业大学学报, 2009,41(11) : 197-200.

[19] 王 霞, 吴加学. 基于小波变换的水沙关系特征分析: 以长江大通站为例. 海洋学研究,2009,27(2):16-22.

[20] 张微微, 李 红, 孙丹峰等. 密云水库上游白河磷浓度时间序列的傅立叶与小波分析. 中国农业科学, 2011, 44 (10) :2060-2069.

[21] 袁礼海,宋建社. 小波变换中的信号边界延拓方法研究. 计算机应用研究, 2006,3:25-27.

[22] 陈伟民. 湖泊生态系统观测方法. 北京: 中国环境科学出版社, 2004 . 